RUB-TPII-55/93, TPR-93-38, November 1993

hep-ph/9312219

\title{
Electric Polarizability of the Nucleon in the Nambu-Jona-Lasinio Model
}

\author{
Emil N. Nikolov ${ }^{1 *}$, Wojciech Broniowski ${ }^{\dagger} \uparrow$, and Klaus Goeke ${ }^{1}$ \\ ${ }^{1}$ Institut für Theoretische Physik II, Ruhr-Universität Bochum \\ D-44780 Bochum, Germany \\ ${ }^{2}$ Institut für Theoretische Physik II, Universität Regensburg \\ D-93040 Regensburg, Germany
}

\begin{abstract}
The electric polarizability of the nucleon is calculated in the soliton approach to the Nambu-Jona-Lasinio model. We analyze the leading- $N_{c}$ contributions, as well as the effects of rotational $1 / N_{c}$ corrections and $\Delta-N$ mass splitting. Our model prediction is substantially reduced compared to other soliton calculations, and is closer to the experimental value.
\end{abstract}

PACS: 12.40.Aa, 11.15.Pg, 11.40.Ha

Typeset using REVTEX

\footnotetext{
* On leave of absence from Institute for Nuclear Research and Nuclear Energy, 1784 Sofia, Bulgaria; DAAD fellow

${ }^{\dagger}$ On leave of absence from Institute of Nuclear Physics, PL-31342 Cracow, Poland;

Alexander von Humboldt fellow

E-mail addresses: emiln or goeke@hadron.tp2.ruhr-uni-bochum.de

broniowski@rphs1.physik.uni-regensburg.de
} 


\section{INTRODUCTION}

Electromagnetic polarizabilities are fundamental properties of hadrons which are manifest in various processes involving two photons [1, 2]. Recent measurements [3] 5] of the electric, $\alpha$, and magnetic, $\beta$, polarizabilities of the nucleon narrowed considerably experimental uncertainties in these observables, and were accompanied by a number of theoretical studies. Attempts to describe $\alpha$ and $\beta$ were made in various approaches, ranging from chiral perturbation theory [6,7] and dispersion relations [8] to chiral soliton models [9 14]. Earlier calculations of $\alpha$ and $\beta$ are reviewed in Ref. [15].

In this paper we calculate the average electric polarizability of the proton and neutron, $\alpha \equiv \frac{1}{2}\left(\alpha_{p}+\alpha_{n}\right)$, using the solitonic approach [16,17] to the Nambu-Jona-Lasinio model [18]. This model has led to quite successful phenomenology [19,20. In particular, baryonic mass relations, magnetic moments, and various form factors are reproduced well within expectations. It is therefore challenging to see if we can also describe two-current observables, such as the polarizability, in the NJL model.

There are two important features in our study which have not been considered in earlier works in soliton models: 1) the role of the Dirac sea effects (this can only be done in a model which has the Dirac sea, such as the NJL model), and 2) inclusion of rotational $1 / N_{c}$-effects. Such effects were recently analyzed for the case of the axial coupling constant $g_{A}$ in Ref. 21] and were more closely examined in Refs. 22, 23.

In the NJL model the only dynamical degrees of freedom are quarks, which occupy valence orbitals, as well as the Dirac sea. We find that the effects of the Dirac sea in our calculation of $\alpha$ are very well reproduced by the first two terms in the gradient expansion, where the first term is the so called "seagull" contribution to $\alpha$, discussed in many previous papers 99,10,12]. Our analysis demonstrates explicitly that the inclusion of the sea-gull in the $\sigma$-model or in the Skyrme model does not violate gauge invariance, as has recently been claimed in Ref. [24]. This is readily seen from the full NJL expression, which is purely dispersive in character (i.e. involves no seagull terms) and manifestly gauge invariant. The seagull term emerges from the full expression when the gradient expansion is performed (see Sec. [II]).

The inclusion of rotational $1 / N_{c}$ effects has very sound phenomenological consequences. As discussed extensively in Ref. [12], chiral soliton models, when treated at the leading- $N_{c}$ level, have problems in predicting electromagnetic polarizabilities correctly. The dominant contribution to the electric polarizability is obtained from pion tail effects, and is proportional to $g_{A}^{2}$. If one insists that the model fits the value of $g_{A}$ (which one should!), then 
the value of $\alpha$ obtained at the leading $N_{c}$ level is roughly a factor of $2-3$ too large, as demonstrated in the $\sigma$-model calculation of Ref. [12]. In the NJL model (as well as in the Skyrmion) the value of $g_{A}$ obtained with various fits has always been notoriously too small at the leading $-N_{c}$ level [25], i.e. of the order $0.7-0.8$. As first noticed in Ref. [21], the inclusion of rotational $1 / N_{c}$ corrections in the NJL model is capable of raising $g_{A}$ to a comfortable value of $\sim 1.2$ [22,23. This is a big correction: it raises $g_{A}^{2}$ by a factor of $2-3$. We calculate analogous $1 / N_{c}$ corrections for the electric polarizability, and find a sizeable contribution. As a result, and after including approximately additional corrections due to the $N-\Delta$ mass splitting [26], we are able to obtain a number which is closer to experiment than in other studies in soliton models [12,14, but still too large. For the typical choice [19,20 of the constituent quark mass $M=420 \mathrm{MeV}$ we obtain $\alpha \simeq 19 \times 10^{-4} \mathrm{fm}^{3}$ compared to the experimental value $\alpha_{\exp }=9.6 \pm 1.8 \pm 2.2 \times 10^{-4} \mathrm{fm}^{3}$ [3] 5 .

In this paper we do not analyze the splitting of the proton and neutron polarizabilities, since it involves a complicated problem of treating the translational zero mode [11]. Also, the magnetic polarizability is not analyzed. It was shown in Ref. [12] that the large- $N_{c}$ approach is not a good starting point to describe this observable. Hence, we concentrate solely on the average proton and neutron electric polarizability, $\alpha$.

The outline of the paper is as follows: In Sec. II we develop the necessary formalism of linear response theory for the NJL model, and derive a basic expression for the electric polarizability. In Sec. III we calculate the leading- $N_{c}$ contribution to $\alpha$, which comes from both valence and sea quark effects. In Sec. IV we calculate the rotational $1 / N_{c}$ corrections to $\alpha$. The valence contribution is calculated exactly, while the sea part is estimated using gradient expansion. Additional corrections, due to $\Delta-N$ mass splitting, are discussed in Sec. V. Sec. VI contains our results and conclusions.

\section{FORMALISM}

Polarizabilities are defined as coefficients in the low-energy expansion of the Compton amplitude [1,2,24]. It has been shown [1,2,24] that the polarizability measured in Compton scattering, $\bar{\alpha}$, can be written as $\bar{\alpha}=\alpha+\Delta \alpha$, where

$$
\alpha=2 \sum_{X \neq N} \frac{\left|\left\langle N\left|D_{z}\right| X\right\rangle\right|^{2}}{E_{N}-E_{X}}, \quad \Delta \alpha=\frac{e^{2}\left\langle r^{2}\right\rangle_{E}}{3 M}+\frac{e^{2}\left(\kappa^{2}+1\right)}{4 M^{3}} .
$$

Here $|N\rangle$ is the nucleon state, and $|X\rangle$ is any intermediate excited state connected by the electric dipole operator $D_{z}$. The first term in the expression for $\Delta \alpha$ is the so called recoil correction, which involves the charge, $e$, the mass, $M$, and the mean squared charge radius 
of the particle, and the second term is the Schwinger scattering term for a particle with anomalous magnetic moment $\kappa$. Our goal is to calculate the dispersive contribution $\alpha$ in Eq. (11). Note that according to Eq. (11), this is equivalent to calculating the coefficient of the second-order energy shift of the nucleon in the constant electric field.

First, we very briefly review the NJL model [16,19]. Our expressions are written in the Euclidean space-time. In the leading- $N_{c}$ treatment the effective action results from taking into account one-quark loops. It contains the quark part, $S_{\text {eff }}^{F}$, the chirally invariant mesonic part, $S_{\text {eff }}^{M}$, and the chiral symmetry breaking part, $S_{\mathrm{eff}}^{\mathrm{br}}$, which provides the pion its mass

$$
\begin{gathered}
S_{\mathrm{eff}}=S_{\mathrm{eff}}^{F}+S_{\mathrm{eff}}^{M}+S_{\mathrm{eff}}^{\mathrm{br}} \\
S_{\mathrm{eff}}^{F}=-\operatorname{Tr} \ln _{\Lambda}\left(i \not \partial-M U^{\gamma_{5}}\right)-\mathrm{vac}, \\
S_{\mathrm{eff}}^{M}=\frac{\mu^{2}}{2} \int d^{4} x\left(\sigma^{2}+\pi^{2}\right)-\mathrm{vac}, \\
S_{\mathrm{eff}}^{\mathrm{br}}=-\frac{m_{0} \mu^{2}}{g} \int d^{4} x \sigma-\mathrm{vac} .
\end{gathered}
$$

The trace is over the quark spinors. The subscript $\Lambda$ reminds of the NJL cut-off (in this paper we use the proper time regulator [27,28]), $m_{0}$ is the current quark mass, and $M$ is the constituent quark mass. The matrix $U^{\gamma_{5}}$ represents the meson hedgehog profile in the non-linear representation

$$
U^{\gamma_{5}}(\boldsymbol{r})=\exp \left(i \gamma_{5} \boldsymbol{\tau} \cdot \widehat{\boldsymbol{r}} \Theta(r)\right)=\frac{1}{f_{\pi}}\left(\sigma_{h}(\boldsymbol{r})+i \gamma_{5} \boldsymbol{\tau} \cdot \boldsymbol{\pi}_{h}(\boldsymbol{r})\right),
$$

where $\Theta(r)$ is the chiral angle, $\sigma_{h}(\boldsymbol{r})=\sigma_{h}(r)$ and $\boldsymbol{\pi}_{h}(\boldsymbol{r})=\widehat{\boldsymbol{r}} \pi_{h}(r)$ are the sigma and pion hedgehog profiles with $\sigma_{h}(r)=f_{\pi} \cos \Theta(r)$ and $\pi_{h}(r)=f_{\pi} \sin \Theta(r), \widehat{\boldsymbol{r}}$ is the radial unit vector, and $\boldsymbol{\tau}$ are the Pauli isospin matrices. The parameters of the model are fixed by reproducing the experimental values of the pion mass, $m_{\pi}$, and the pion decay constant, $f_{\pi}$, leaving the quark-meson coupling constant $g$, or, equivalently, the constituent quark mass $M=g f_{\pi}$ as the only free parameter [19].

Solitonic solutions to the NJL model are found by selfconsistently solving the coupled Euler-Lagrange equations for the profile function $\Theta$ and the Dirac spinors $q_{\mathrm{n}}$, which satisfy the Dirac equation

$$
H q_{\mathrm{n}}=\left(-i \boldsymbol{\nabla} \cdot \boldsymbol{\alpha}+\beta M U^{\gamma_{5}}\right) q_{\mathrm{n}}=\epsilon_{\mathrm{n}} q_{\mathrm{n}}
$$

In the presence of the the electromagnetic field $A^{\mu}$, the minimal substitution $\partial^{\mu} \rightarrow \partial^{\mu}+i e Q A^{\mu}$, with the quark charge $Q=\left(\frac{1}{2 N_{c}}+\frac{1}{2} \tau^{3}\right)$, leads to the following gauged effective action 


$$
S_{\text {eff }}^{A}=-\operatorname{Tr} \ln _{\Lambda}\left(i \not \partial-e Q A-M U^{\gamma_{5}}\right)-\text { vac. }
$$

The hedgehog solution brakes the spin, $J$, and isospin, $I$, symmetries of the lagrangian, preserving the grand spin $G=J+I$ symmetry. To restore good quantum numbers we use the semiclassical "cranking" projection scheme [29,30]. Introducing the collective rotation matrix $R(t)=B \exp \left(i \frac{\boldsymbol{\Omega} \cdot \boldsymbol{\tau}}{2} t\right)$ in the usual way and transforming the quark fields and meson profiles to the isorotating frame [29,30] we obtain

$$
\widetilde{S}_{\text {eff }}^{A}=-\operatorname{Tr} \ln _{\Lambda}\left(i \not \partial-e B^{\dagger} Q B A-i \beta \frac{\Omega \cdot \tau}{2}-M U^{\gamma_{5}}\right)-\text { vac. }
$$

It is convenient to introduce the notation $B^{\dagger} \tau^{3} B=\boldsymbol{c} \cdot \boldsymbol{\tau}$, where $c^{a}$ is an element of a Wigner $D$-matrix, $c^{a}=D_{a 3}^{1}[31$. Then the quark electric charge in the isorotating frame can be written as

$$
B^{\dagger} Q B=\frac{1}{2 N_{c}}+\frac{\boldsymbol{c} \cdot \boldsymbol{\tau}}{2}
$$

The action (9) describes the system which is perturbed by small perturbations: electromagnetic, and cranking. From now on our method will follow very closely Ref. [31]. The idea is the following: we have found the soliton, and now we want to calculate its linear response to external perturbations. The perturbation operators consist of products of two parts: intrinsic, which act on the internal structure of the state, and collective, which act on collective coordinates. For the cranking perturbation, $i \beta \frac{\boldsymbol{\Omega} \cdot \boldsymbol{\tau}}{2}$, the collective operator is $\boldsymbol{\Omega}$, and $i \beta \boldsymbol{\tau} / 2$ is the intrinsic operator. Similarly, the electromagnetic perturbation has a collective part (vector $\boldsymbol{c}$ in Eq. (10)) and an intrinsic part. Also, the nucleon state is a product of a collective and an intrinsic part 29 31. Formal perturbative expansion of Eq. (9) to $n$-th order in small parameters involves expressions of the general form

$$
\begin{aligned}
\kappa_{i_{1}, \ldots, i_{n}}= & \left\langle\text { coll }\left|\mathcal{V}_{i_{1}}^{\text {coll }} \mathcal{V}_{i_{2}}^{\text {coll }} \ldots \mathcal{V}_{i_{n}}^{\text {coll }}\right| \text { coll }\right\rangle \\
& \int d^{4} x_{1} \ldots d^{4} x_{n}\langle H| \mathcal{V}_{i_{1}}^{\text {intr }}\left(x_{1}\right) G\left(x_{1}, x_{2}\right) \mathcal{V}_{i_{2}}^{\text {intr }}\left(x_{2}\right) \\
& G\left(x_{2}, x_{3}\right) \ldots \mathcal{V}_{i_{n-1}}^{\text {intr }}\left(x_{n-1}\right) G\left(x_{n-1}, x_{n}\right) \mathcal{V}_{i_{n}}^{\text {intr }}\left(x_{n}\right) G\left(x_{n}, x_{1}\right)|H\rangle,
\end{aligned}
$$

where $|c o l l\rangle$ is the baryon collective wave function, $|H\rangle$ is the intrinsic hedgehog state, $\mathcal{V}_{i_{k}}$ is the perturbation of the $k^{\text {th }}$ type, and $G$ is the quark Feynman propagator in the background meson field, which in the spectral representation has the form

$$
G(x, y)=\left(\Theta\left(x_{4}-y_{4}\right) \sum_{n \in \text { unocc. }} e^{-\epsilon_{n}\left(x_{4}-y_{4}\right)}-\Theta\left(y_{4}-x_{4}\right) \sum_{n \in \text { occ. }} e^{-\epsilon_{n}\left(x_{4}-y_{4}\right)}\right) q_{\mathrm{n}}(\boldsymbol{x}) q_{\mathrm{n}}^{\dagger}(\boldsymbol{y}),
$$

where occ. denotes all occupied states, i.e. the valence as well as the Dirac sea states and unocc. denotes the unoccupied positive energy states.

Examples of application of Eq. (11) are given in Ref. 31. 


\section{LEADING- $N_{C}$ CONTRIBUTIONS TO $\alpha$}

It was shown in Ref. [12] that the leading contribution to the electric polarizability of the nucleon scales as $N_{c}$. Our first task is to obtain this leading contribution for $\alpha$ in the NJL model. We denote it by $\alpha^{(0)}$. Since the cranking frequency $\Omega \sim 1 / N_{c}$, the leading- $N_{c}$ contribution is obtained by expanding the effective action (9) to second order in the electric field, and to zero order in $\Omega$. From Eq. (10) we notice, that for the leading $N_{c}$ contribution we have to keep only the isovector part of the charge, since the isoscalar part is one power of $N_{c}$ down.

We now take $A^{4}=E z, \boldsymbol{A}=0$, and expand Eq. (8) to second order in $E$ and zero order in $\Omega$. We obtain the formula of the form (11)

$$
\begin{aligned}
\alpha^{(0)} & =-\frac{1}{T} e^{2}\left\langle N\left|\operatorname{Tr}\left(\frac{\boldsymbol{c} \cdot \boldsymbol{\tau}}{2} x_{3} G(x, y) \frac{\boldsymbol{c} \cdot \boldsymbol{\tau}}{2} y_{3} G(y, x)\right)\right| N\right\rangle \\
& =\frac{1}{2} N_{c} e^{2}\left\langle N\left|c^{a} c^{b}\right| N\right\rangle \sum_{\substack{m \in \text { occ. } \\
n \in \text { unocc. }}} \frac{\left\langle m\left|\tau^{a} z\right| n\right\rangle\left\langle n\left|\tau^{b} z\right| m\right\rangle}{\epsilon_{n}-\epsilon_{m}}
\end{aligned}
$$

where $|N\rangle$ is the collective nucleon state, $T$ is a large time interval, over which the time integrations are carried, and $|n\rangle$ and $\epsilon_{n}$ denote the eigenstates and eigenvalues of the Dirac hamiltonian (7). We can now split this formula into the valence and sea parts. The sea contribution carries the cut-off function, and the vacuum contribution has to be subtracted. Finally, using $\left\langle N\left|c^{a} c^{b}\right| N\right\rangle=\frac{1}{3} \delta^{a b}$, we get the following formulas for the leading $N_{c}$ valence and sea contributions to the electric polarizability of the nucleon

$$
\begin{gathered}
\alpha_{\mathrm{val}}^{(0)}=\frac{N_{c}}{6} e^{2} \sum_{n \neq \mathrm{val}} \frac{\left\langle\operatorname{val}\left|\tau^{a} z\right| n\right\rangle\left\langle n\left|\tau^{a} z\right| \mathrm{val}\right\rangle}{\epsilon_{n}-\epsilon_{\mathrm{val}}} \\
\alpha_{\mathrm{sea}}^{(0)}=\frac{N_{c}}{6} e^{2} \sum_{n \neq m} R\left(\epsilon_{m}, \epsilon_{n}, \Lambda\right)\left\langle m\left|\tau^{a} z\right| n\right\rangle\left\langle n\left|\tau^{a} z\right| m\right\rangle-\mathrm{vac},
\end{gathered}
$$

where $R\left(\epsilon_{m}, \epsilon_{n}, \Lambda\right)$ is the proper time regularization function

$$
R\left(\epsilon_{m}, \epsilon_{n}, \Lambda\right)=-\frac{1}{4 \sqrt{\pi}} \int_{\Lambda^{-2}}^{\infty} \frac{d s}{s^{3 / 2}}\left[\frac{e^{-s \epsilon_{m}^{2}}-e^{-s \epsilon_{n}^{2}}}{\epsilon_{m}^{2}-\epsilon_{n}^{2}}+s \frac{\epsilon_{m} e^{-s \epsilon_{m}^{2}}+\epsilon_{n} s e^{-s \epsilon_{n}^{2}}}{\epsilon_{m}+\epsilon_{n}}\right],
$$

with $\Lambda$ denoting the (proper time) cut-off parameter. The value of $\Lambda$ is fit for any $M$ to reproduce the experimental value of the pion coupling constant $f_{\pi}$ using the amplitude of the weak pion decay [19]. The subtracted vacuum contribution in Eq. (15) has the same

structure as the first term, but the free quark states and eigenvalues are used. It may be immediately verified that both the valence and sea contributions to $\alpha^{(0)}$ scale as $N_{c}$ [12]. 
In the NJL model, predictions depending on the cut-off are somewhat ambiguous. As we shall see, however, in our case $\alpha_{\text {sea }}^{(0)}$ is dominated by the lowest term in the gradient expansion, which is independent of the cut-off.

The numerical results for the valence, $\alpha_{\text {val }}^{(0)}$, and Dirac sea, $\alpha_{\text {sea }}^{(0)}$, parts of $\alpha^{(0)}$ are plotted in Fig. 1 as a function of the constituent quark mass $M$. Both contributions decrease with increasing $M$, the valence curve has much larger slope for smaller $M$ due to the fast increasing energy gap between the valence orbital and the positive continuum. For the optimum value [19,20 of $M=420 \mathrm{MeV}$, we obtain $\alpha^{(0)}=16 \times 10^{-4} \mathrm{fm}^{3}$. The sea contributes to $\alpha^{(0)} 65 \%$, and dominates over the valence part, which gives 35\%. Since the Dirac sea effects describe the physics of the pion cloud, this result is in qualitative agreement with the results of other models [32, 12, 14].

We have also performed the gradient expansion of $\alpha_{\text {sea }}^{(0)}$ up to first two terms following the scheme of Refs. 33 35]. This provides a link to the $\sigma$-model [36,12 as well as an independent check for our numerical procedure in the case of large size solitons. The leading-order term in the gradient expansion of $\alpha_{\text {sea }}^{(0)}$ gives us exactly the seagull term of the $\sigma$-model

$$
\alpha_{\text {seagull }}=\frac{8 \pi e^{2}}{9} \int d r r^{4}\left(\pi_{h}(r)\right)^{2} .
$$

Note that this seagull term arises in the NJL model from purely dispersive Dirac sea effects (cf. Eq. (15)). Recently, the inclusion of the seagull term in soliton models such as the $\sigma$-model or the Skyrmion has been questioned [24]. The NJL model provides a simple interpretation of how Eq. (17) arises in the $\sigma$-model, and clears the doubts of Ref. [24]. Numerically the seagull term gives more than $80 \%$ of the total sea contribution.

Looking at absolute numbers it may seem striking that our value of the seagull contribution, $\sim 9 \times 10^{-4} \mathrm{fm}^{3}$, and the $\sigma$-model [12] value, $\sim 30 \times 10^{-4} \mathrm{fm}^{3}$ differ so drastically. The explanation lies in the large difference in the values of the axial coupling constant at the leading $N_{c}$ level. Our model predicts $g_{A}^{(0)} \sim 0.75$, while in the $\sigma$-model $g_{A}^{(0)}=1.42$ [30]. The asymptotic pion tail, which gives the dominant contribution in (17), is proportional to $g_{A}^{(0)}$ and since the pion field enters quadratically in (17) the large effect follows. We will come back to the question of $g_{A}$ in the next section.

It is interesting to investigate in some greater detail how well the gradient expansion works for the electric polarizability. In Fig. 2 we compare the results of the gradient expansion with the full calculation of $\alpha_{\text {sea }}^{(0)}$ for large-size solitons parameterized by the profile function involving a size parameter $R$

$$
\Theta(r)=-2 \operatorname{atan}\left(\frac{R^{2}}{r^{2}}\left(1+m_{\pi} r\right) e^{-m_{\pi} r}\right) .
$$


With increasing size of the soliton the result of the gradient expansion for $\alpha_{\text {sea }}^{(0)}$ up to first two terms converges to the full result very well. At $R=2 \mathrm{fm}$ the difference is only $2 \%$.

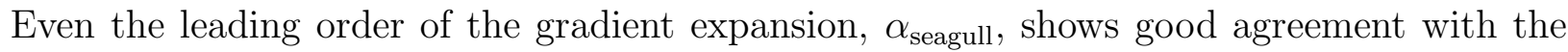
full result, reproducing it up to $90 \%$ at $R=2 \mathrm{fm}$.

\section{ROTATIONAL CORRECTIONS TO $\alpha$}

Now we turn to the problem of the next-to-leading order corrections in the $1 / N_{c}$ expansion of the electric polarizability $\alpha$. As already mentioned above, the value of $g_{A}$ in chiral soliton models is crucial for the results for $\alpha$. The prediction for $g_{A}$ in the NJL model (as well as in the Skyrme model) at the leading- $N_{c}$ level, denoted by $g_{A}^{(0)}$, turns out to be much too small, $\sim 0.7-0.8$ [25], and no parameter fits or extensions of the model were able to raise this value. Recently, Ref. [21] proposed how to include rotational $1 / N_{c}$ corrections to $g_{A}$ in the NJL model. A correct and detailed study of this effect has been presented in Refs. [22,23, 37]. The nature of these corrections is very easy to understand, for instance from the point of view of the formalism of Ref. [31]. At the leading- $N_{c}$ level the expression for $g_{A}$ involves a sum of matrix elements of the axial operator in the quark single-particle states [25]. However, in the presence of cranking the hedgehog state is perturbed, and we have a situation where one of the perturbations is the axial vector current, and the other one is the cranking. The effect discussed in Refs. [21,23, 22] is associated with the noncommutativity of the collective parts of the corresponding operators (the cranking and the axial vector current operators in this case). An analogous effect arises for the case of $\alpha$, as we discuss below.

Since the cranking operator scales as $1 / N_{c}$ (it involves the cranking frequency $\Omega \sim 1 / N_{c}$ ) the correction is suppressed by one power of $N_{c}$. This correction to the axial vector coupling constant, denoted by $g_{A}^{(1)}$, has the valence part and the sea part. The numerical value for $g_{A}^{(1)}$ is about 0.5 [21 23], and raises the total value of $g_{A}$ to a comfortable number. Of course, the described $1 / N_{c}$ corrections are not the only possible ones, but they seem to be particularly important.

We have explained before that the small value of $g_{A}^{(0)}$ reduces the value of the seagull contribution to the polarizability, which is a desired effect. Since roatational $1 / N_{c}$ corrections raise significantly the value of $g_{A}$, we have also to examine how analogous effects influence the electric polarizability. The $1 / N_{c}$ rotational corrections to $\alpha$ are obtained by expanding the effective action (9) to second order in $E$ and first order in $\boldsymbol{\Omega}$. Thus, we have formally the case of the third-order perturbation theory. We get expression of the generic form (11), 
with all possible time-orderings of collective operators.

The relevant collective matrix elements for the present case have the form

$$
\left\langle N\left|c^{a} \Omega^{b} c^{c}\right| N\right\rangle=\frac{1}{3 \mathcal{J}} \epsilon^{a b c},
$$

where $\mathcal{J}$ is the moment of inertia. Finally, after straightforward algebra, we obtain for the valence contribution

$$
\begin{aligned}
& \alpha_{\text {val }}^{(1)}=-\frac{i N_{c} e^{2} \epsilon^{a b c}}{12 \mathcal{J}} \\
& \times\left[\sum_{\substack{n>0, n \neq \text { val } \\
m>0, m \neq \text { val }}} \frac{\left\langle\operatorname{val}\left|\tau^{a} z\right| n\right\rangle\left\langle n\left|\tau^{b}\right| m\right\rangle\left\langle m\left|\tau^{c} z\right| \mathrm{val}\right\rangle}{\left(\epsilon_{n}-\epsilon_{\mathrm{val}}\right)\left(\epsilon_{m}-\epsilon_{\mathrm{val}}\right)}-\sum_{\substack{n<0 \\
m<0}} \frac{\left\langle\operatorname{val}\left|\tau^{a} z\right| n\right\rangle\left\langle n\left|\tau^{b}\right| m\right\rangle\left\langle m\left|\tau^{c} z\right| \mathrm{val}\right\rangle}{\left(\epsilon_{n}-\epsilon_{\mathrm{val}}\right)\left(\epsilon_{m}-\epsilon_{\mathrm{val}}\right)}\right. \\
& \left.-2 \sum_{\substack{n>0, n \neq \mathrm{val} \\
m<0}} \frac{\left\langle\operatorname{val}\left|\tau^{a} z\right| n\right\rangle\left\langle n\left|\tau^{b} z\right| m\right\rangle\left\langle m\left|\tau^{c}\right| \mathrm{val}\right\rangle}{\left(\epsilon_{n}-\epsilon_{\mathrm{val}}\right)\left(\epsilon_{n}-\epsilon_{m}\right)}+2 \sum_{\substack{n<0 \\
m>0}} \frac{\left\langle\mathrm{val}\left|\tau^{a} z\right| n\right\rangle\left\langle n\left|\tau^{b} z\right| m\right\rangle\left\langle m\left|\tau^{c}\right| \mathrm{val}\right\rangle}{\left(\epsilon_{n}-\epsilon_{\mathrm{val}}\right)\left(\epsilon_{n}-\epsilon_{m}\right)}\right],
\end{aligned}
$$

and for the sea contribution

$$
\begin{aligned}
& \alpha_{\text {sea }}^{(1)}=-\frac{i N_{c} e^{2} \epsilon^{a b c}}{12 \mathcal{J}} \\
& \times\left[\sum_{\substack{k<0 \\
m, n>0}} \frac{\left\langle k\left|\tau^{a} z\right| n\right\rangle\left\langle n\left|\tau^{b}\right| m\right\rangle\left\langle m\left|\tau^{c} z\right| k\right\rangle}{\left(\epsilon_{k}-\epsilon_{m}\right)\left(\epsilon_{k}-\epsilon_{n}\right)}+\sum_{\substack{k>0 \\
m, n<0}} \frac{\left\langle k\left|\tau^{a} z\right| n\right\rangle\left\langle n\left|\tau^{b}\right| m\right\rangle\left\langle m\left|\tau^{c} z\right| k\right\rangle}{\left(\epsilon_{k}-\epsilon_{m}\right)\left(\epsilon_{k}-\epsilon_{n}\right)}\right]-\text { vac. }
\end{aligned}
$$

In the above expressions notation $n<0$ means the level with $\epsilon_{n}<0$, etc. Recalling that $\mathcal{J} \sim N_{c}$, it can be verified that $\alpha^{(1)}$ is of order $N_{c}^{0}$, while the leading contribution to $\alpha^{(0)}$ was of order $N_{c}$.

The numerical value for $\alpha_{\text {val }}^{(1)}$ is obtained directly from Eq. (20). It involves a double summation over quark levels, and computer time involved is similar to the calculation of, say, the moment of inertia. Our results are shown in Fig. 1. The value of $\alpha_{\text {val }}^{(1)}$ is about $3 \times 10^{-4} \mathrm{fm}^{3}$ for $M=420 \mathrm{MeV}$, so the effect is about $60 \%$ of the leading- $N_{c}$ valence contribution $\alpha_{\text {val }}^{(0)}$. The decrease of $\alpha_{\text {val }}^{(1)}$ with increasing $M$ is similar to the behavior of the leading- $N_{c}$ valence contribution $\alpha_{\mathrm{val}}^{(0)}$.

Now we turn to the sea contribution $\alpha_{\text {sea }}^{(1)}$. This contribution in principle may involve a cut-off, similar to other observables calculated in the NJL model. However, since Eq. (21) involves a computer-time-consuming triple sum over quark states, we approximate $\alpha_{\text {sea }}^{(1)}$ by the leading term in the gradient expansion. As a bonus, we get the nice feature that the result is independent of the cut-off, exactly as in the case of the leading- $N_{c}$ contribution $\alpha_{\text {seagull. }}$. In the present case the gradient expansion technique involves some tricks [38], so we sketch the method in some greater detail. The first step is to rewrite Eq. (21) in a form 
involving unconstrained sums, i.e. sums over all quark levels. This can be achieved using the identity true for any real numbers $a, b$ and $c$

$$
\begin{aligned}
& \int \frac{d \omega_{1} d \omega_{2} d \omega_{3}}{(2 \pi)^{3}} \frac{1}{\omega_{1}-i a} \frac{1}{\omega_{1}-i b} \frac{1}{\omega_{1}-i c} \frac{1}{\omega_{1}-\omega_{2}-i \eta} \frac{1}{\omega_{1}-\omega_{3}-i \eta} \\
& =-i \frac{1}{a-b} \frac{1}{a-c} \Theta(-a) \Theta(b) \Theta(c) .
\end{aligned}
$$

With help of Eq. (22) we can replace the constrained sums in Eq. (21) by sums over all indices. For instance

$$
\begin{aligned}
& \sum_{\substack{k<0 \\
m, n>0}} \frac{\left\langle k\left|\tau^{a} z\right| n\right\rangle\left\langle n\left|\tau^{b}\right| m\right\rangle\left\langle m\left|\tau^{c} z\right| k\right\rangle}{\left(\epsilon_{k}-\epsilon_{m}\right)\left(\epsilon_{k}-\epsilon_{n}\right)} \\
& =i \sum_{k, m, n}\left\langle k\left|\tau^{a} z\right| n\right\rangle\left\langle n\left|\tau^{b}\right| m\right\rangle\left\langle m\left|\tau^{c} z\right| k\right\rangle \int \frac{d \omega_{1} d \omega_{2} d \omega_{3}}{(2 \pi)^{3}} \frac{1}{\omega_{1}-i \epsilon_{k}} \frac{1}{\omega_{1}-i \epsilon_{m}} \frac{1}{\omega_{1}-i \epsilon_{n}} \\
& \times\left(P \frac{1}{\omega_{1}-\omega_{2}}+i \pi \delta\left(\omega_{1}-\omega_{2}\right)\right)\left(P \frac{1}{\omega_{1}-\omega_{3}}+i \pi \delta\left(\omega_{1}-\omega_{3}\right)\right)
\end{aligned}
$$

where $P$ denotes the principal value integral. Introducing the trace over the quark spinors $\operatorname{Tr}$ we can now rewrite Eq. (21) as

$$
\begin{aligned}
\alpha_{\text {sea }}^{(1)} & =\frac{i \epsilon^{a b c} e^{2}}{12 \mathcal{J}} \int \frac{d \omega_{1} d \omega_{2}}{(2 \pi)^{2}} P \frac{1}{\omega_{1}-\omega_{2}} \\
& \times \operatorname{Tr}\left[\tau^{a} z \frac{1}{\omega_{1}-i H} \tau^{b} \frac{1}{\omega_{2}-i H} \tau^{c} z \frac{1}{\omega_{1}-i H}+\tau^{a} z \frac{1}{\omega_{1}-i H} \tau^{b} \frac{1}{\omega_{1}-i H} \tau^{c} z \frac{1}{\omega_{2}-i H}\right]-\text { vac. }
\end{aligned}
$$

Standard gradient expansion techniques may now be used to expand the functional trace in Eq. (24) [33]. The lowest order term (denoted by lowest), contains no gradients, and we obtain

$$
\begin{aligned}
& \alpha_{\text {sea,lowest }}^{(1)}=-\frac{32 N_{c} M^{2}}{3 \mathcal{J} f_{\pi}^{2}} \int \frac{d^{3} k}{(2 \pi)^{3}} \int \frac{d \omega_{1} d \omega_{2}}{(2 \pi)^{2}} P \frac{1}{\omega_{1}-\omega_{2}} \frac{\left(2 \omega_{1}+\omega_{2}\right)}{\left(\omega_{1}^{2}+k^{2}+M^{2}\right)^{2}\left(\omega_{2}^{2}+k^{2}+M^{2}\right)} \\
& \quad \times \int d^{3} r z^{2}\left(\pi_{h}(r)\right)^{2} \\
& \quad=\frac{N_{c} M}{16 \pi \mathcal{J} f_{\pi}^{2}} \alpha_{\text {seagull }},
\end{aligned}
$$

where $\alpha_{\text {seagull }}$ is given in Eq. (17).

For $M=420 \mathrm{MeV}$ the moment of inertia has the value $\mathcal{J}=1.1 \mathrm{fm}$, and we obtain for the proportionality factor in the last equality in Eq. (25) the value 0.54. The numerical results for $\alpha_{\text {sea,lowest }}^{(1)}$ are plotted in Fig. 1. The contribution increases with increasing constituent quark mass $M$. This is due to the decrease of the moment of inertia with $M$. Guided by our experience from gradient-expanding the leading- $N_{c}$ contribution (Sec. III), we hope that 
also in the present case the lowest term in the gradient expansion almost saturates the value of $\alpha_{\text {sea }}^{(1)}$.

At the end of this section we would like to present an interesting observation. The formula analogous to Eq. (25) for the case of $g_{A}$ has the form [37]

$$
g_{A, \text { sea,lowest }}^{(1)}=\frac{N_{c} M}{32 \pi \mathcal{J} f_{\pi}^{2}} g_{A, \text { sea,lowest }}^{(0)} \text {. }
$$

Note that the coefficient between the $1 / N_{c}$ correction and the leading- $N_{c}$ term in Eq. (26) is exactly twice the analogous coefficient in Eq. (25). This is not a coincidence. In the chiral limit, $m_{\pi} \rightarrow 0$, the sea contribution to $\alpha$ is divergent as $1 / m_{\pi}$,6, 12,26]. This divergent piece is proportional to $g_{A}^{2}$, and this result is a general, model independent feature of the chiral limit. Therefore, one has $\alpha_{\text {sea }}=\frac{\gamma}{m_{\pi}} g_{A}^{2}$, where $\gamma$ is a numerical constant. Hence, if in a model one corrects $g_{A}$ by including $1 / N_{c}$ terms, analogous correction should come out in the calculation of $1 / N_{c}$ corrections to $\alpha$. Consider the formal case of solitons with a very large size $R$. In that limit, $g_{A}$ consists only of the sea contribution, which in turn is saturated by

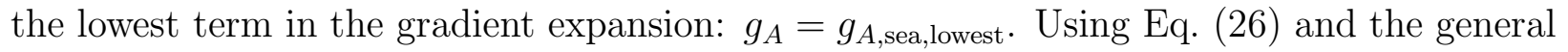
considerations discussed above, we obtain in the double limit of $m_{\pi} \rightarrow 0$ and $R \rightarrow \infty$ the following result

$$
\begin{aligned}
\alpha_{\text {sea,lowest }} & =\frac{\gamma}{m_{\pi}} g_{A}^{2}=\frac{\gamma}{m_{\pi}}\left(g_{A, \text { sea,lowest }}^{(0)}+g_{A, \text { sea,lowest }}^{(1)}\right)^{2}+\mathcal{O}\left(1 / N_{c}^{2}\right) \\
& =\alpha_{\text {seagull }}+\frac{N_{c} M}{16 \pi \mathcal{J} f_{\pi}^{2}} \alpha_{\text {seagull }}+\mathcal{O}\left(1 / N_{c}^{2}\right)
\end{aligned}
$$

The first term in the last line of is our $\alpha_{\text {sea,lowest }}^{(0)}$, the second term is $\alpha_{\text {sea,lowest }}^{(1)}$, with exactly the same factor as in Eq. (25). This shows that our method of including rotational $1 / N_{c}$ corrections is consistent with chiral physics.

Analogous consistency conditions have to hold for other observables which behave as $g_{A}^{2} / m_{\pi}$, e.g. the isovector magnetic mean squared radius of the nucleon.

Another digression concerns the structure of the rotational $1 / N_{c}$ corrections. Collective operators which arise in evaluating corrections to $g_{A}$ or the isovector magnetic moment have the same structure as collective operators entering at leading- $N_{c}$ level. Formally, this is because $\left[J^{a}, D_{b c}^{1}\right]=i \epsilon^{a b d} D_{d c}^{1}$. Thus, ratios of these observables (also for non-diagonal matrix elements) for various members of the flavor $\mathrm{SU}(2)$ multiplet (i.e. for $N$ and $\Delta$ ) are preserved at the $1 / N_{c}$ level, and obtain corrections starting at the $1 / N_{c}^{2}$ level. This complies to the consistency condition of Ref. [39]. 


\section{EFFECTS OF THE $\Delta-N$ MASS SPLITTING}

Before comparing our results to data, we note that there is another very important effect which has not been included, namely the $N-\Delta$ mass splitting. As discussed in Ref. [12,26], in the limit $N_{c} \rightarrow \infty$ our perturbative approach has the nucleon and the $\Delta$ degenerate in mass. At the hadronic level, the seagull term results from diagrams with intermediate pionnucleon or pion- $\Delta$ states. Because of the $N-\Delta$ degeneracy, the contribution of the pion- $\Delta$ states is strongly overestimated in the hedgehog treatment. One may crudely include the effect of $N-\Delta$ mass splitting by reducing the seagull contribution by about $30 \%$ [12,26]. A consistent treatment of this effect would require the use of a better collective quantization method, but this does not seem feasible for the NJL model. As shown in Section III more than $80 \%$ of the sea contribution $\alpha_{\text {sea }}^{(0)}$ is carried by the seagull term. Therefore, as suggested in Refs. [12,26], we simply multiply the sea contribution by a factor of $2 / 3$ to mimic the effect of $N-\Delta$ mass splitting.

Since $\alpha_{\text {sea,lowest }}^{(1)}$ is proportional to $\alpha_{\text {seagull }}$, we expect that it obtains analogous corrections from $\Delta-N$ splitting as the leading- $N_{c}$ part. Therefore we also multiply it by a factor of $2 / 3$ before comparing to data.

\section{FINAL RESULT AND CONCLUSION}

Our numerical results are collected in Figs. 1,3. They are plotted as a function of the constituent quark mass $M$. Figure 1 shows the sea and valence contributions to the leading$N_{c}$ nucleon electric polarizability $\alpha^{(0)}$ as well as to the rotational $1 / N_{c}$ correction $\alpha^{(1)}$. The total polarizability is presented on Fig. 3 compared to the experimental value. The dashed line corresponds to $\alpha^{\prime}=\alpha_{\text {sea }}^{(0)}+\alpha_{\text {val }}^{(0)}+\alpha_{\text {sea,lowest }}^{(1)}+\alpha_{\text {val }}^{(1)}$. The full line shows the total $\alpha$, crudely corrected for the effects of the $N-\Delta$ mass splitting by multiplying the sea contributions by a factor of $2 / 3$ (Sec. $\mathrm{V}): \alpha=\frac{2}{3} \alpha_{\text {sea }}^{(0)}+\alpha_{\text {val }}^{(0)}+\frac{2}{3} \alpha_{\text {sea,lowest }}^{(1)}+\alpha_{\text {val }}^{(1)}$. For values of $M$ in the physically relevant range 400-450 MeV the changes of $\alpha$ with $M$ are small. Choosing for $M=420 \mathrm{MeV}$ (value which gives good agreement for other nucleon observables [19,20]) we obtain for the total nucleon electric polarizability the value $\alpha=19 \times 10^{-4} \mathrm{fm}^{3}$, with $\frac{2}{3} \alpha_{\text {sea }}^{(0)}, \alpha_{\text {val }}^{(0)}, \frac{2}{3} \alpha_{\text {sea,lowest }}^{(1)}$, and $\alpha_{\text {val }}^{(1)}$ contributing $36 \%, 29 \%, 17 \%$, and $17 \%$ respectively. Our result is still too large compared to the experimental number $\alpha_{\exp }=9.6 \pm 1.8 \pm 2.2 \times 10^{-4} \mathrm{fm}^{3}[3]$. Keeping in mind the approximate nature of the model and techniques used, our model prediction is within expectations.

Note, that in our calculation, after including the rotational $1 / N_{c}$ effects, we have the 
correct value of $g_{A}$. This is not the case of other models [9,10,13], and apparent agreement of $\alpha$ with experiment in these models results from having too low $g_{A}$.

The main points of this paper can be summarized as follows:

1) Dirac sea effects dominate the electric polarizability of the nucleon. In the gradient expansion the sea effects are dominated by the seagull contribution. This explains how this term arises in other models which do not have the explicit Dirac sea.

2) We have calculated rotational $1 / N_{c}$ corrections, and found that they are important and of expected size.

3) We have crudely estimated the $N-\Delta$ splitting effects.

4) With all above effects included, numerical predictions of the NJL model are better than in other soliton models (note that we have the correct value of $g_{A}$ !) but are still about a factor of 2 too large compared to experiment. This indicates that the NJL soliton is too "soft" and other effects are still needed in order to describe the polarizability properly.

5) Calculation of the magnetic polarizability, or the proton-neutron splitting of the electric polarizability would require a much greater effort in the NJL model than in the $\sigma$-model calculation of Ref. [11,12]. This is because of the necessity to subtract zero-modes.

The authors are grateful to Pavel Pobylitsa for helpful comments. This work has been supported in part by the Polish State Committee for Scientific Research, grants 2.0204.91.01 and 2.0091.91.01, and by the Bulgarian National Science Foundation, contract $\Phi-32$. 


\section{REFERENCES}

[1] V. A. Petrun'kin, Sov. J. Part. Nuc. 12 (1981) 278.

[2] J. L. Friar, in Proc. Workshop on electron-nucleus scattering, EIPC, Marciana, Italy, edited by A. Fabrocini et al. (World Scientific, Singapore, 1989).

[3] F. Federspiel et al., Phys. Rev. Lett. 67 (1991) 1511.

[4] A. Zieger et al., Phys. Lett. B278 (1992) 34.

[5] J. Schmiedmayer, P. Riehs, J. Harvey, and N. Hill, Phys. Rev. Lett. 66 (1991) 1015.

[6] V. Bernard, N. Kaiser, and U.-G. Meissner, Phys. Rev. Lett. 67 (1991) 1515; Nucl. Phys. B373 (1992) 346.

[7] M. N. Butler and M. J. Savage, Phys. Lett. B294 (1992) 369.

[8] A. I. L'vov, Phys. Lett. B304 (1993) 29.

[9] M. Chemtob, Nucl. Phys. A473 (1987) 613.

[10] N. N. Scoccola and W. Weise, Phys. Lett. B232 (1989) 287; Nucl. Phys. A517 (1990) 495.

[11] W. Broniowski, M. K. Banerjee, and T. D. Cohen, Phys. Lett. B283 (1992) 22.

[12] W. Broniowski and T. D. Cohen, Phys. Rev. D47 (1993) 299.

[13] B. Schwesinger, Phys. Lett. B298 (1993) 17.

[14] B. Golli and R. Sraka, Phys. Lett. B312 (1993) 24.

[15] G. M. G. Dattoli and D. Prosperi, Lett. Nuovo Cimento 19 (1977) 601.

[16] Th. Meissner, F. Grümmer and K. Goeke, Phys. Lett. B227 (1989) 296.

[17] H. Reinhardt and R. Wünsch, Phys. Lett. B215 (1988) 577.

[18] Y. Nambu and G. Jona-Lasinio, Phys. Rev. 122 (1961) 345.

[19] Th. Meissner and K. Goeke, Nucl. Phys. A524 (1991) 719.

[20] A. Z. Górski, C. V. Christov, F. Grümmer and K. Goeke, Ruhr-Univ. Bochum preprint No. RUB-TPII-56-93 (1993).

[21] M. Wakamatsu and T. Watabe, Phys. Lett. B312 (1993) 184. 
[22] C. V. Christov, K. Goeke, P. V. Pobylitsa, M. Polyakov, T. Watabe, and M. Wakamatsu, Ruhr-Univ. Bochum preprint No. RUB-TPII-53-93 (1993).

[23] A. Blotz, M. Praszałowicz, and K. Goeke, Phys. Lett. B317 (1993) 195.

[24] A. I. L’vov, Univ. Mainz preprint No. MKPH-T-93-6 (1993).

[25] Th. Meissner and K. Goeke, Z. Phys. A339 (1991) 513.

[26] T. D. Cohen and W. Broniowski, Phys. Lett. B292 (1992) 5.

[27] J. Schwinger, Phys. Rev. 82 (1951) 664.

[28] D. I. Dyakonov and V. Y. Petrov, JETP Lett. 43 (1986) 57.

[29] G. S. Adkins, C. R. Nappi, and E. Witten, Nucl. Phys. B228 (1983) 552.

[30] T. D. Cohen and W. Broniowski, Phys. Rev. D34 (1986) 3472.

[31] W. Broniowski and T. D. Cohen, Phys. Rev. D47 (1993) 313.

[32] R. Weiner and W. Weise, Phys. Lett. B159 (1985) 85.

[33] I. J. R. Aitchison and C. M. Frazer, Phys. Lett. B146 (1984) 63; Phys. Rev. D31 (1985) 2605; Phys. Rev. D32 (1985) 2190.

[34] E. Ruiz Arriola, Phys. Lett. B253 (1991) 430.

[35] C. Schüren, E. Ruiz Arriola, and K. Goeke, Nucl. Phys. A547 (1992) 612.

[36] M. C. Birse and M. K. Banerjee, Phys. Lett. B136 (1984) 284; Phys. Rev. D31 (1985) 118.

[37] A. Blotz, M. Praszałowicz, and K. Goeke, Ruhr-Univ. Bochum preprint No. RUB-TPII41-93 (1993).

[38] We thank Pavel Pobylitsa for inventing these tricks.

[39] R. Dashen and A. V. Manohar, UC, San Diego preprint No. UCSD/PTH 93-16, 93-18 (1993). 


\section{FIGURE CAPTIONS}

Fig. 1.

The electric polarizability of the nucleon, $\alpha$, as a function of the constituent quark mass $M$. Leading $N_{c}$ Dirac sea contribution $\alpha_{\text {sea }}^{(0)}$ (dashed line); leading $N_{c}$ valence contribution $\alpha_{\text {val }}^{(0)}$ (dash-dotted line); rotational $1 / N_{c}$ correction to the sea contribution $\alpha_{\text {sea,lowest }}^{(1)}$ (dotted line) and to the valence one $\alpha_{\text {val }}^{(1)}$ (dash-dot-dot).

Fig. 2.

The ratio of the gradient expanded electric nucleon polarizability to the full leading- $N_{c}$ $\alpha^{(0)}=\alpha_{\text {sea }}^{(0)}+\alpha_{\text {val }}^{(0)}$ for different soliton sizes $R$. The dash-dotted line represents the leading order in the gradient expansion $\alpha_{\text {seagull }}$ and the dashed line the seagull plus the next term in the gradient expanded $\alpha$.

\section{Fig. 3.}

The total electric polarizability of the nucleon, $\alpha$, as a function of the constituent quark mass $M$. The dashed line shows the full $\alpha^{\prime}=\alpha_{\text {sea }}^{(0)}+\alpha_{\text {val }}^{(0)}+\alpha_{\text {sea,lowest }}^{(1)}+\alpha_{\text {val }}^{(1)}$ and the full line $\alpha=\frac{2}{3} \alpha_{\text {sea }}^{(0)}+\alpha_{\text {val }}^{(0)}+\frac{2}{3} \alpha_{\text {sea,leading }}^{(1)}+\alpha_{\text {val }}^{(1)}$, where the factor $\frac{2}{3}$ in front of $\alpha_{\text {sea }}^{(i)}$ crudely corrects for the overestimation of the $\Delta$ contribution (Sec. $\mathrm{V}$ ). 
$\alpha\left[10^{-4} \mathrm{fm}^{3}\right]$

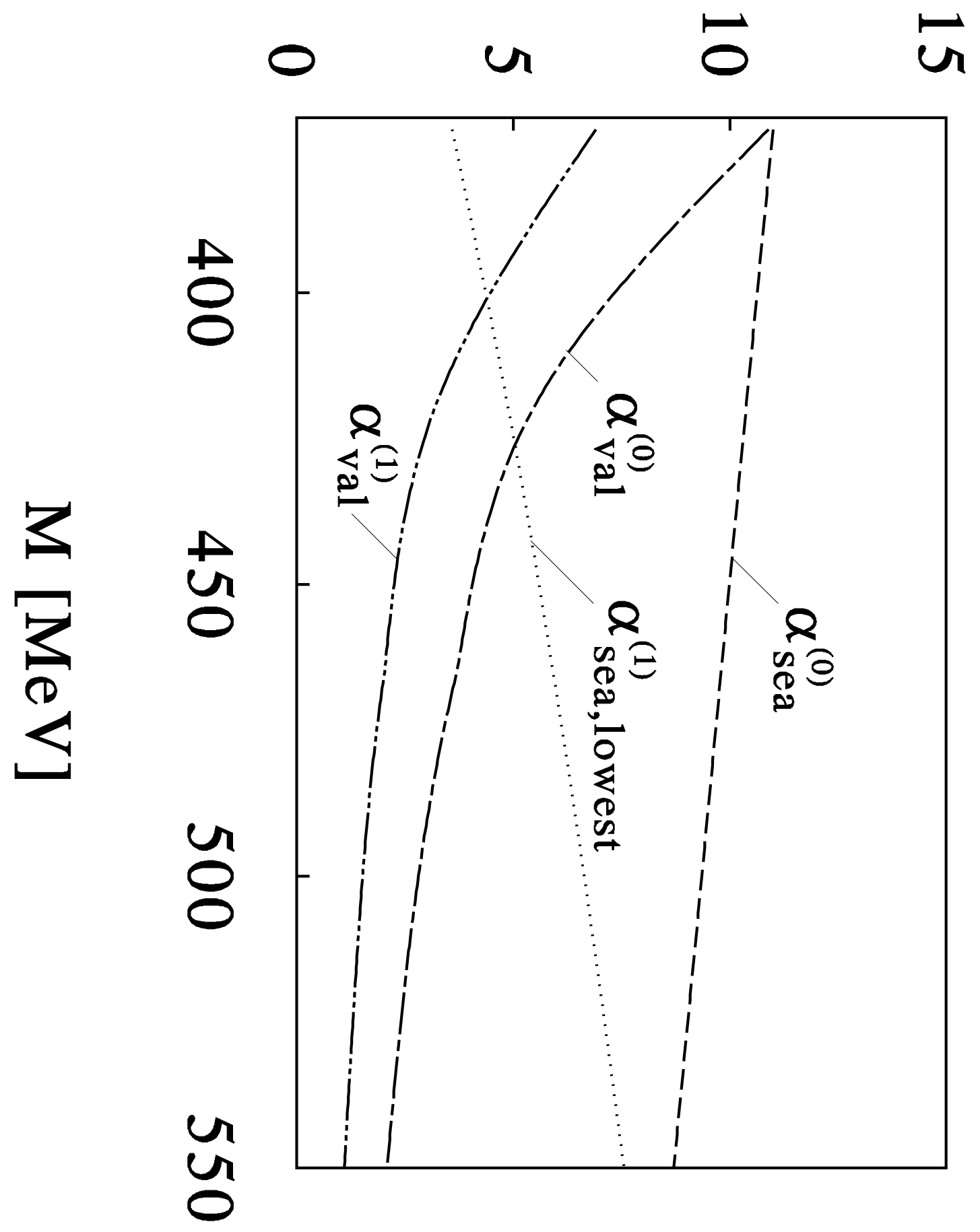




$$
\alpha_{\mathrm{GE}}^{(0)} / \alpha^{(0)}[\%]
$$

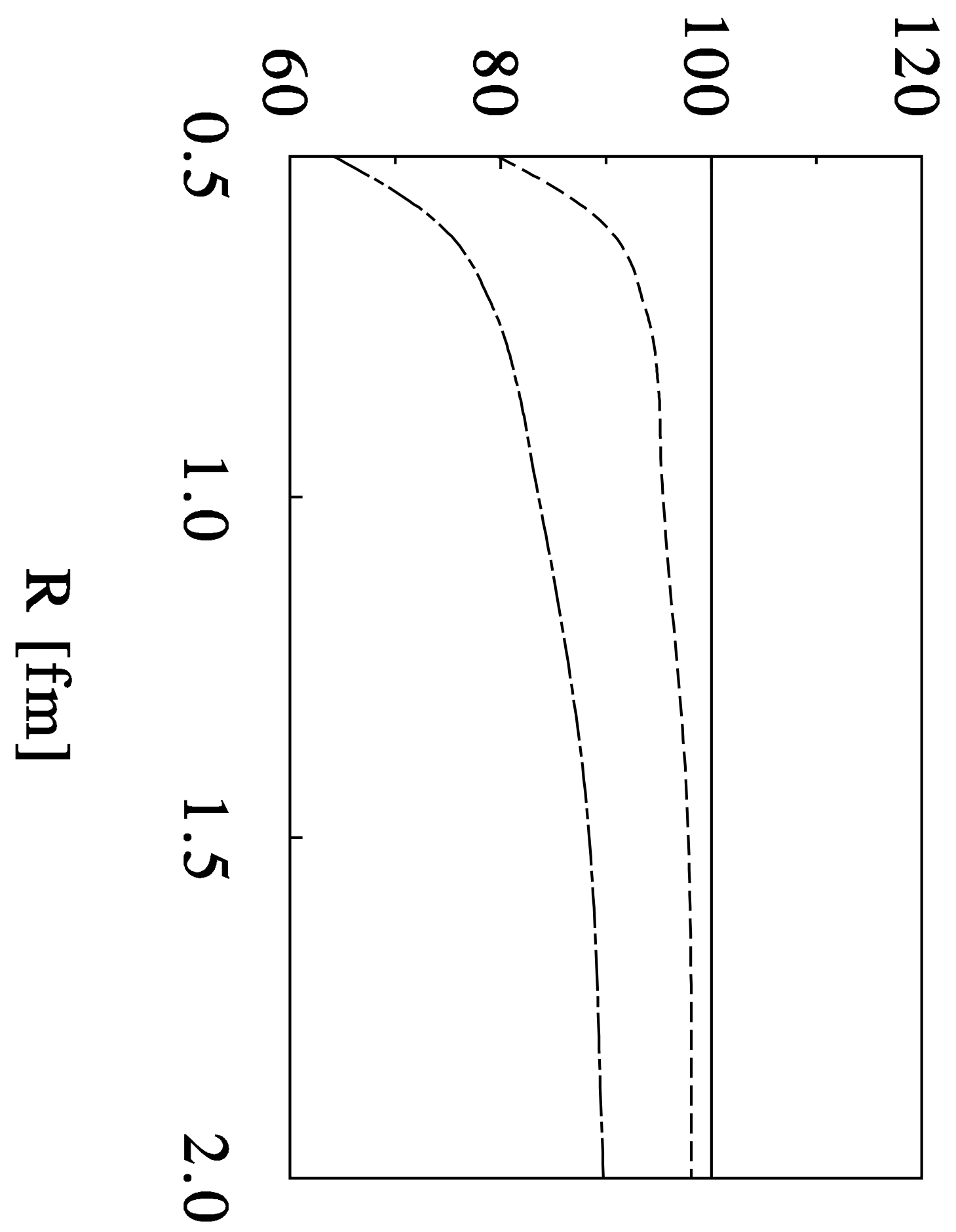


$\alpha\left[10^{-4} \mathrm{fm}^{3}\right]$

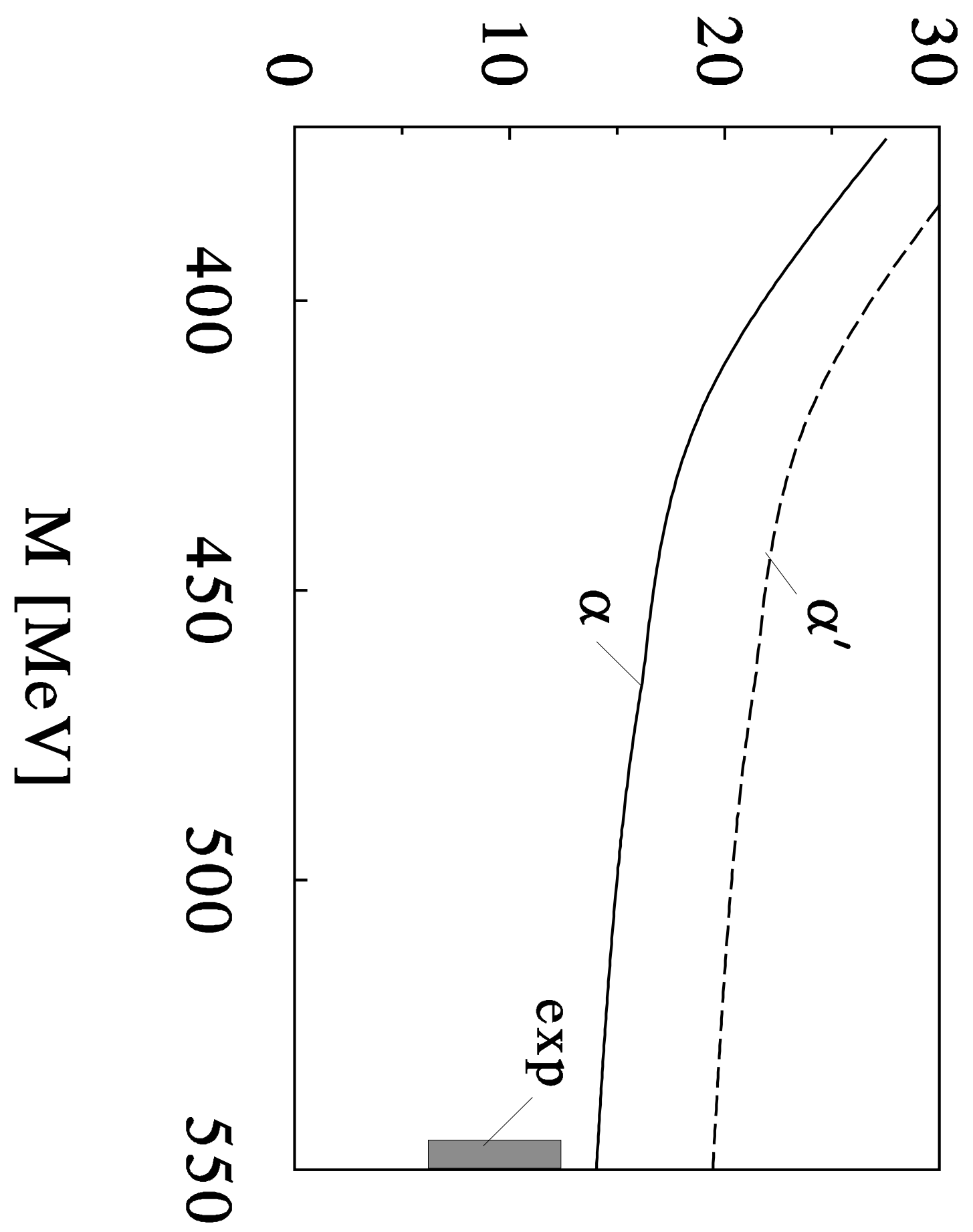

